\title{
Using a Champion-Oriented Mindset to Overcome the Challenges of Graduate School
}

Andrea G. Marshall ${ }^{1 *}$, Caroline B. Palavicino-Maggio ${ }^{2 *}$, Kit Neikirk ${ }^{3}$, Zer Vue ${ }^{1}$, Heather Beasley ${ }^{1}$, Edgar Garza-Lopez ${ }^{4}$, Sandra Murray ${ }^{5}$, Denise Martinez ${ }^{6}$, Jamaine Davis $^{7}$, Haysetta Shuler, ${ }^{8,9 \#, ~ E l s i e ~ C . ~}$ Spencer ${ }^{10 \#}$, Derrick Morton ${ }^{11 \#}$, Antentor Hinton, $\mathrm{Jr}^{1,4 \#}$

Author Information

${ }^{1}$ Department of Molecular Physiology and Biophysics, Vanderbilt University, Nashville, TN

${ }^{2}$ Department of Neurobiology, Harvard Medical School, Boston, MA

${ }^{3}$ Department of Biology, University of Hawaii, Hilo, HI

${ }^{4}$ Hinton and Garza Lopez Family Consulting Company, Iowa City, IA

${ }^{5}$ Department of Cell Biology, University of Pittsburgh School of Medicine, Pittsburgh, PA

${ }^{6}$ Department of Family Medicine, University of Iowa, Iowa City, IA

7 Department of Biochemistry, Cancer Biology, Neuroscience, Pharmacology, Meharry Medical College, Nashville, TN

${ }^{8}$ Department of Biological Sciences, Winston-Salem State University, Winston-Salem, NC

${ }^{9}$ Shuler Consulting, Winston-Salem, NC

${ }^{10}$ Teachers College, Columbia University, New York, NY, USA

${ }^{11}$ Department of Biological Sciences, University of Southern California, Los Angeles, CA

*These authors share co-first authorship

\#These authors share co-senior authorship

Co-Corresponding Author: antentor.o.hinton.jr@ vanderbilt.edu

Co-Corresponding Author: mortond@usc.edu

\begin{abstract}
Despite efforts to increase diversity, a glaring underrepresentation of minorities (URM) persists in the fields of science, technology, engineering, and mathematics (STEM). Graduate school can be a stressful step in the STEM pipeline, especially for students previously unaware of the structure and challenges of post-graduate education. To promote successful minority participation in STEM and prepare prospective students for the impending challenges of graduate school, we developed a workshop based on the mentoring and fostering of a champion-oriented mindset entitled, "The Trials and Tribulations of Graduate School: How Do You Make an Impact?". We administered the workshop to a cohort of university undergraduates and conducted pre- and post-workshop surveys to measure students' perceived need for instruction on specific workshop topics. The results suggest that the workshop was well received by the students and provided information that they considered helpful to help navigate the graduate school process.
\end{abstract}

Key Words: graduate school, champion mindset, URM, personal statement, interviewing 


\section{Introduction:}

2 Despite many institutional initiatives and programs aimed to increase the presence of underrepresented

3 minorities (URMs) in science, technology, engineering, and mathematics (STEM), URM participation in

4 STEM fields still remains disproportionately low, in part because support programs often have limited

5 availability and therefore fail to reach these pool of students [1, 2]. Here, we define URMs as Black or

6 African Americans, Latinx or Hispanic Americans, American Indians, Alaskan Natives, Native

7 Hawaiians, and Native Pacific Islanders [3]. According to the National Science Foundation (NSF) and the

8 National Science Board (NSB), there is a critical need to bolster the presence and retention of URMs in

9 STEM. Diversity in STEM is important because multiformity leads to more impactful research, improved

10 problem solving skills, decreased turnover rates [4], and promotes elevated innovation [3]. Moreover, the

11 number of STEM occupations is expected to increase at almost twice the rate of overall job growth in the

12 U.S. [1, 2]. This can potentially lead to even greater racial disparities unless intentional efforts are

13 increased to promote and sustain URM participation in STEM. As part of these efforts, we believe that

14 faculty should take an active role by providing mentorship, support, and advocacy for URM students

15 pursuing STEM careers.

17 Graduate- and post-graduate level education is required for many STEM occupations. Beyond the daily

18 stresses of graduate school, URMs face additional challenges that can hinder their productivity and deter

19 them from pursuing or obtaining a career in STEM, including microaggressions [5], imposter syndrome

$20[3,5,6]$, awfulizing and demoralization [7], and lack of or decreased access to mentors [4]. Importantly,

21 affirmative mentoring [8-10], access to career development resources, and self-efficacy [11-13] are

22 correlated with positive academic and professional outcomes in STEM [14, 15] [16]. Some organizations

23 including the National Institutes of Health (NIH) have programs like the Maximizing Access to Research

24 Careers (MARC) and Research Initiative for Scientific Enhancement (RISE) training grants, that seek to

25 enhance and promote URM presence in STEM by providing financial support as well as research,

26 training, professional development, and mentoring activities to help students prepare for graduate school 
27 (https://www.nigms.nih.gov/training/marc/pages/ustarawards.aspx). However, these programs are not

28 available at every university and the number of opportunities available for each training grant is limited,

29 thus curtailing the overall reach of their impact. To help close this gap and promote graduate school

30 preparedness among as many URM students in STEM as possible, we developed a workshop based on the

31 framework of intentional mentoring. Participants in the workshop are trained how to increase their self-

32 efficacy skills by adopting a "champion's mindset" to overcome the hurdles that they will face in the

33 STEM pipeline.

\section{Framework for the Workshop}

36 Here, we present the content of the workshop regarding what undergraduate students should expect in

37 graduate school and how they can maximize their chances of success in a graduate program. Students are

38 surveyed before the workshop and after its completion.

\section{The Champion's Mindset}

41 In the workshop, participants are presented a model in which intelligence is viewed as either static and

42 unchangeable (fixed mindset) or flexible with room for development (champion's mindset; Figure 1)[17,

43 18]. Individuals with a fixed mindset tend to be fragile and avoid challenges because they view them as

44 threats that might reveal inherent personal deficiencies. These individuals also interpret expenditure of

45 effort as a sign of low capacity and setbacks as evidence of low ability [19]. By contrast, individuals with

46 a champion's mindset innately seek out challenges because they perceive themselves as able to conquer

47 them, create opportunities for improvement, and better hone their abilities [19]. Research has shown that

48 students at all levels who approach learning with a champion's mindset learn more, achieve more, and

49 perform better academically than students who approach learning with a static mindset [19]. Therefore,

50 our workshop emphasizes the importance of adopting a champion's mindset as they enter a graduate

\section{1 program.}


54 Early exposure to STEM experiences can significantly influence a student's motivation and preparedness

55 to pursue a career in STEM [3, 4, 20]. Therefore, students who participated in the workshop were

56 encouraged to seek opportunities to conduct research prior to applying or attending graduate school

57 through programs such as the NIH's T34 Bridges to the Baccalaureate Research Training Program,

58 Summer Research Opportunity Program, or Research Experiences for Undergraduates [3]. The workshop

59 emphasized that research experiences can occur both within and outside the student's home institution.

60 However, it is important to underscore that because many minority-serving institutions (MSI's) lack the

61 research infrastructure and funds to support such programs, it is critical that students be made aware of

62 the existence of these programs at other schools.

Mentors Are a Necessity

65 Another key element highlighted in the workshop is the power and importance of mentors. Effective mentoring is personal and reciprocal in nature. It helps the mentee achieve their goals, and provides

67 psychosocial, professional, and career support [7]. Importantly, mentoring can mitigate the pitfalls that

68 URMs in graduate programs commonly face, including burnout, minority stress, imposter syndrome,

69 demoralization, and awfulization [3, 5-7]. This support is accomplished through intentionality and

70 purpose: the mentors and mentees are deliberate in getting to know one another, communicate openly and

71 frequently, continually reflect on the mentor-mentee relationship, and work in unison to achieve the

72 mentee's goals. This is implemented by utilizing tools such as mentoring contracts and individual

73 development plans (IDP's) [7]. In our workshop, students are provided with practical advice on choosing

74 mentors who are genuinely committed to their success, invested in their long-term development, and able

75 to focus on their strengths while addressing their weaknesses in a proactive manner. Students are also

76 advised to seek multiple mentors with different strengths, rather than limiting themselves to only one

77 mentor. We understand that the process of identifying effective mentors can take time, but we strongly

78 encourage students to look for role models as well, such as people outside of the institution who are 
79 successful practitioners in an area (or areas) of interest to them and/or those who have overcome adversity

80 themselves. Even when students lack a mentor, they can still use proactive (rather than reactive)

81 development options to effectively prepare for graduate school. For example, students can read about

82 their role model's journey and learn what steps that person took to become successful including past

83 grants they were awarded and various public or community engagements they were involved in during

84 their careers. This will allow a mentee to gauge how well that individual lead, inspired, and cultivated

85 other future URMs in STEM fields.

\section{Distinguishing Oneself in Graduate School Applications}

88 Specific application requirements vary by university and program. Typically applications require official

89 transcripts detailing the applicant's overall grade point average (GPA) and possibly the GPA within the

90 subject area of the applicant's major; class ranking; a condensed personal statement; graduate record

91 examination (GRE) scores (although some programs waive this requirement); multiple letters of

92 recommendation; and a resume or curriculum vitae (CV). Understanding the relevance a CV plays into

93 the application process is critical. The CV should indicate all honors and awards received by the applicant

94 (e.g., fellowships, scholarships, travel awards), relevant job experience (e.g., internships, research,

95 teaching), participation in campus organizations (e.g., society memberships, sports activities, club

96 memberships), community service, scholarship, and leadership activities. The minimum GPA requirement

97 for many Ph.D. programs is 3.0 or equivalent to a B-. If their GPA is lower than 3.0, the applicant may

98 consider retaking courses (if applicable), applying to a post-baccalaureate program, or pursue a master's

99 degree before applying to a Ph.D. program. This suggestion will not be frowned upon by admissions

100 committees but will highlight the willingness and determination that an applicant has in pursuing a

101 doctoral degree in STEM. GRE scores within the $75^{\text {th }}$ percentile are acceptable for most schools;

102 however, scores in the $90^{\text {th }}$ percentile or above are desirable and typically accepted by all schools. 
104 The personal statement should be the applicant's "story" or positionality that draws in the reader and

105 motivates them to meet the applicant in person. Therefore, word choice is critical. Effective personal

106 statements usually include an exciting hook or angle that distinguishes the author from other applicants. It

107 explains why the individual wants to pursue a Ph.D. degree in general and what they intend to do with

108 that degree from that institution should they be accepted. In general, all applicants to competitive schools

109 have exceptional GPAs and GRE scores, so the personal statement should include information that makes

110 the candidate stand out among other qualified individuals. Things that stand out are involvement in

111 extracurricular activities, volunteer or community work, and publications. The personal statement should

112 also explain why the applicant chose to apply to the specific school and program. This is an opportunity

113 for admissions committee members to see how much you understand the inner workings of their

114 institutions and how well aligned you are to their mission. Furthermore, it is acceptable to discuss both

115 strengths and weaknesses in the personal statement, however, the applicant should be careful to avoid

116 coming across as overconfident or unqualified.

118 Students should be forward-thinking when identifying individuals from whom they may ask for a letter of 119 recommendation. Someone who knows the applicant well (e.g., their strengths and weaknesses, struggles 120 and triumphs, and work ethic), can likely write a more personalized and ultimately effective letter. Hence, 121 it is generally not recommended for students to ask for a letter of recommendation from a professor who

122 they have only seen in a classroom filled with hundreds of other students. The best way to obtain effective

123 letters of recommendation is to establish and maintain good relationships with professors, mentors, and

124 research associates before applying to graduate programs. In this scenario, when applying to graduate

125 programs, students can more readily request letters from people who know them well and can speak to

126 specific qualities that make them an ideal candidate. It is important to emphasize that time is of critical

127 essence when requesting a letter of recommendation. An applicant typically should give the author a

128 minimum of 4-6-weeks' notice before the submission deadline when requesting a letter of

129 recommendation. Phrasing is key when asking a potential recommender. It should not be assumed that a 
130 recommender will be willing to provide one or able to within the allotted time. But if they agree, which

131 they normally do, students should for a strongly written, positive letter of recommendation addressed to

132 their program of choice. The student should then pay close attention to the nuances of the answer because

133 a recommendation can be positive, negative, or neutral. Once a person agrees to write a strong, affirming

134 letter, the student should provide them with a copy of transcripts, a reminder of pertinent shared

135 experiences, an outline of their goals, and a CV detailing all relevant research experience, internships,

136 leadership activities, awards, and achievements that can be referenced in the letter as needed.

138 Before applying to graduate school, and after the application process, it is important for students to reflect 139 on what might be beneficial for their mental health. This is a critical step that often gets overlooked prior 140 and during the application process. For example, students may find it useful to explore their personality

141 type or identify activities that restore their energy by using tools such as the Big Five Personality Test, the

142 Myers-Briggs Type Indicator, or the Five Love Languages. Students should expect graduate school to be 143 challenging, not just intellectually but in all aspects of their life. Hence, the better they know themselves

144 and their unique learning styles, the more prepared they will be to overcome and address the challenges of 145 pursuing a graduate education head-on.

147 Interviewing Prospective Graduate Programs

148 Applicants should remember that they are assessing graduate programs just as much as graduate programs 149 are assessing them. They should therefore proactively research the institutions that will be their home 150 away from home for the next five to six years. This research should also include academic departments,

151 the clinical affiliations of the institution (if any), and the cities and neighborhoods surrounding the

152 institution. Some questions to consider during this process include: What types of communities exist that

153 might be a good fit for the applicant? Are current URM graduate students happy or satisfied with the

154 program and school? Are resources available to accommodate and support the specific needs of the

155 applicant? What is the faculty to student ratio? These questions are important to consider because 
156 graduate school can be a prolonged season of both personal and professional trials, therefore, applicants

157 should use their interviews with prospective graduate programs as opportunities to gauge whether each

158 program is right for them. They should truly ask themselves whether that institution and its culture and

159 environment could be a place in which the individual may develop a sense of community, which will in

160 turn help them to succeed.

162 The interview phase is another process of the graduate school process that students need to be prepared to

163 successfully undergo. Regardless of whether the interview is on-site or virtual, the applicant must be

164 enthusiastic and convey curiosity, competence, and courtesy. Unless otherwise stated, the expected attire

165 for in-person interviews is generally semi-formal but comfortable enough to endure the several hours

166 required to complete the interview process, which is also referred to as "running the gauntlet". Before the

167 day of the interview, applicants should familiarize themselves with the faculty in the department, their

168 research interests and accolades, and how the applicant will fit into that environment. Applicants should

169 also identify three to five potential mentors at that institution who they would like to meet during their

170 visit and potentially work with for the duration of graduate school.

172 The Structure of Graduate School

173 Before starting graduate school, students should rest, recharge, refresh, and engage in a champion's

174 mindset. Table 1 shows the general structure and suggested goals for each year of graduate school.

175 Typically, the first two years are the most challenging, during which students usually complete most of

176 their required academic coursework (typically at a faster pace than undergraduate courses). They rotate

177 through laboratories, often shadowing senior researchers, principal investigators, and faculty and select

178 one in which to complete their graduate research, and complete their qualifying exam. Throughout these

179 early years, students should be proactive in getting to know their professors and working together with

180 their cohort to excel in courses. Because balancing coursework, relationship building, and laboratory

181 rotations is difficult, it is crucial to engage in self-reflection and stress management activities at this stage 
182 of their academic career. Moreover, it is not uncommon for relationship dynamics with family and friends

183 to change during these years, as graduate school is often more taxing than undergraduate studies. Students

184 and their families have to understand that social events and familial commitments will need to be adjusted

185 due to the student's schedule and workload. For many students, the first two years of graduate school are

186 a transition period during which they shift from mostly following instructions to making their own

187 informed decisions.

189 Another challenge in the graduate school phase is the qualifying exam (QE), an oral exam that determines

190 whether or not students will be allowed to continue in their graduate program after completion of their

191 first two years. This exam is a requirement for all Ph.D. candidates and assesses whether a student is

192 academically prepared for the following years of instruction. Generally, this exam involves an

193 examination committee comprised of three faculty who will ask questions on your proposed research

194 topic then make suggestions and recommendations to strengthen or clarify your research focus. In

195 preparation for this exam, students should be diligent in determining what the exam requires, its format,

196 and schedule sufficient time to study. The conclusion of the QE will result in a committee's suggestion of

197 pass or fail. Most programs have strict rules for what constitutes a pass, conditional pass, or failure, and

198 how many times the exam may be re-taken before a student who fails to pass is dismissed from the

199 program. Students should prioritize QE preparation and allow for ample study time. They should also

200 become familiar with the specific QE rules and content according to their particular program and school

201 by attending similar venues such as doctoral defenses, which are generally open to the public.

203 After successfully passing the QE, the student begins year three of the graduate program. During this year

204 students should consider immersing themselves in career development and grant writing opportunities at

205 their home institution and at subject-relevant organizations. During year four, student should focus on

206 writing research manuscripts and establishing networking collaborations, which is critical because many

207 programs require at least one first-author publication for graduation. Throughout and beyond year five, 
students should consider their next career steps. With guidance from their mentors, they should apply for their next position at least nine to 12 months in advance of when they expect to graduate. This will allow

210 them sufficient time to find a suitable position that aligns with their career goals and to learn any

211 additional skills or experience that may be required for the position. Throughout these years, a student

212 should be expanding their dissertation, which is based on the research topic they used during their QE.

213 Writing each chapter can take months, even years depending on the topic and encapsulates a format that is

214 informative to the reader. It should include research questions, methodology, research framework,

215 discussion, findings, and a conclusion that answers your research questions based on your findings and

216 analyses. In parallel, the process of writing and defending a dissertation or thesis can be daunting, so

217 students should also allow time to correctly format and complete their dissertation by the deadlines set by

218 their school.

Methods:

221 We administered our 90-minute virtual workshop entitled "The Trials and Tribulations of Graduate

222 School: How to Make an Impact" to 24 undergraduate students at Winston-Salem State University (a

223 historically Black public university) from the general student population. This study was submitted to the

224 Kaiser Permanente review board under the title "Promoting Engagement in science for underrepresented

225 Ethnic and Racial minorities (P.E.E.R)," and was approved and classified as not human subjects research.

226 The participants completed questionnaires before and after the workshop (see Table 2) to gauge their

227 expectations and satisfaction regarding the workshop. These were either anonymous paper surveys or an

228 emailed electronic survey. All respondents were informed of the purpose of these surveys, and surveys

229 were on a voluntary basis and independently performed to avoid peer pressure. Respondents that

230 consented to the research returned surveys discreetly in an envelope and/or in an anonymous online form.

231 We summarized the data from the questionnaires using box and whisker plots in which the red centerline

232 denotes the median, and error bars denote the standard error. Individual values are represented by circles.

233 We analyzed the raw data with nonparametric tests for comparison within paired samples. We also used 
234 Wilcoxon matched-pairs and signed-rank tests to determine differences between measures. The p-values

235 less than 0.05 indicated statistical significance and NS were non-significant.

\section{Results:}

\section{Student Responses and Observations}

239 Responses to the pre-workshop questionnaires suggested that students initially did not see much value in

240 the workshop (Figure 2, Figure 6, and Figure 7, pre-test). Whether their low expectations were due to a

241 lack of exposure or a lack of mentorship is not clear. However, at the conclusion of the workshop, scores

242 on the post-workshop questionnaire rose an average of 5.8 points on a scale of one to 10 , with 10

243 indicating the most favorable response (Figure 2, Figure 3, and Figure 4). These results suggest that,

244 although the students were initially unenthusiastic about participating in the workshop or lacked an

245 understanding of the graduate school process, they ultimately found it to be informative and worthwhile

246 (Figure 2A, post-test). Prior to the workshop, students reported having little knowledge of the

247 characteristics of graduate school (Figure 2B) and feeling unprepared for the application process (Figure

248 2C). Amidst their uncertainties, the students still did not think the workshop would be beneficial (Figure

249 2D), emphasizing the need for more "preparedness training". This training refers to graduate school-

250 specific information (e.g., the application process, interviews, mentoring) or rather efforts to convince

251 students that graduate school is attainable with the proper preparation. It is possible that the students did

252 not initially see the benefit of the workshop because they felt graduate school in general was beyond their

253 grasp, thus making the workshop seem irrelevant to their goals, even if it did provide useful information.

254 The students also did not expect the workshop to provide much help and clarity with the documents and

255 experiences vital to the graduate school application process. Despite these low expectations, responses to

256 the post-training questionnaire indicated that the students felt the information presented in the workshop

257 empowered them to draft their personal statements (Figure 3A), seek letters of recommendation (Figure

258 3B), increase their confidence to apply to graduate school (Figure 3C), improve their GRE preparation, 
259 (Figure 3D) increase their networking skills (Figure 3E), and understand the value of research

260 experiences, such as summer internships (Figure 3F). The post-training questionnaire results also

261 indicated that the students felt the workshop was beneficial for their time management skills (Figure 4A),

262 their ability to approach interview questions (Figure 4B), their understanding of the role of support teams

263 (Figure 4C), their communication skills (Figure 4D), and their ability to identify and improve their

264 learning styles (Figure 4E).

\section{Discussion:}

267 Collectively, the data from the questionnaires highlight the need for more career development

268 opportunities, such as the workshop conducted in this paper. Although initial enthusiasm for this type of

269 programming may be low, students can still benefit. Early exposure, mentoring, and incorporation of a

270 champion's mindset are all strategies that motivate and improve academic outcomes for URM students

$271[14][3,4,7-13,15-20]$. Though our sample size of surveyed students was small, our results suggest that

272 many undergraduate URM students will also benefit from career development resources that aim to

273 increase the influx and retention of URMs into the STEM pipeline, especially for those at minority-

274 serving institutions with low retention rates. Success is shown to be more prevalent when students are

275 empowered and equipped with the proper knowledge, tools, and strategies to overcome the overwhelming

276 challenges of graduate school.

\section{Limitations}

278 Due to the small number of participants, caution should be exercised in generalizing the results of this

279 study. We also recognize that our study participants likely do not represent all STEM areas, as only those

280 with a special interest in biology might have volunteered to participate. Moreover, participants may have

281 changed their behavior after the workshop only because they felt obliged to fulfil expectations, rather than

282 a true intent to pursue a career in STEM. Additional questions might have allowed a more comprehensive

283 perspective of the students' feedback. 
286 A PowerPoint presentation of the workshop is available in English and Spanish formats. Survey data may

287 be made available upon reasonable written request.

289 Abbreviations

290 CV - Curriculum Vitae

291 GPA - Grade Point Average

292 GRE - Graduate Record Examinations

293 IDP - Individual Development Plan

294 MARC - Maximizing Access to Research Careers

295 MSI - Minority Serving Institution

296 NIH - National Institute of Health

297 NSB - National Science Board

298 NSF - National Science Foundation

299 QE - Qualifying Exam

300 RISE - Research Initiative for Scientific Enhancement

301 SROP - Summer Research Opportunity Program

302 REU - Research Experiences for Undergraduates

303 STEM - Science, Technology, Engineering, and Mathematics

304 URM - Underrepresented Minority

\section{References}

1. Arbeit, C., Yamaner, M.I., National Center for Science and Engineering Statistics (NCSES), Trends for Graduate Student Enrollment and Postdoctoral Appointments in Science, Engineering, and Health Fields at U.S. Academic Institutions between 2017 and 2019. 2021: Alexandria, VA: National Science Foundation.

$3112 . \quad$ National Science Board, Vision 2030. 2020: Washington, D.C. 
3. Hinton, A.O., Jr., et al., Patching the Leaks: Revitalizing and Reimagining the STEM Pipeline.

$313 \quad$ Cell, 2020. 183(3): 568-575.

314 4. Stelter, R.L., J.B. Kupersmidt, and K.N. Stump, Establishing effective STEM mentoring

315 relationships through mentor training. Ann N Y Acad Sci, 2021. 1483(1): 224-243.

5. Marshall, A., et al., Responding and navigating racialized microaggressions in STEM. Pathog 317 Dis, 2021. 79(5).

6. Hinton, A.O., Jr., et al., Mentoring minority trainees: Minorities in academia face specific challenges that mentors should address to instill confidence. EMBO Rep, 2020. 21(10): p. e51269.

7. Shuler, H., et al., Intentional mentoring: maximizing the impact of underrepresented future 322 scientists in the 21st century. Pathog Dis, 2021. 79(6).

8. Gordon, D.M., et al., Mentoring urban Black Middle-School Male Students: Implications for

9. Anderson, G.N., Dey, E.L., Gray, M., and Thomas, G., Mentors and proteges: The influence of faculty mentoring on undergraduates academic achievement, in Association for the Study of Higher Education. 1995: Orlando, Florida.

10. Thompson, L.A. and L. Kelly-Vance, The impact of mentoring on academic achievement of at-

11. DeFreitas, S.C., Bravo, Antonio, Jr, The Influence of Involvement with Faculty and Mentoring on

12. Honicke, T. and J. Broadbent, The influence of academic self-efficacy on academic performance:

13. Turner, E.A., M. Chandler, and R.W. Heffer, The Influence of Parenting Styles, Achievement

14. Strayhorn, T.L., Factors Influencing Black Males' Preparation for College and Success in STEM

15. Green, A. and D. Sanderson, The Roots of STEM Achievement: An Analysis of Persistence and

16. Kricorian, K., et al., Factors influencing participation of underrepresented students in STEM

17. Dweck, C.S., Motivational processes affecting learning. American Psychologist, 1986. 41: 1040-

18. Dweck, C.S., Mindset: The new psychology of success. 2006, New York, NY: Random House. 
19. Rattan, A., et al., Leveraging Mindsets to Promote Academic Achievement: Policy

20. Hernandez, P.R., Schultz, P. W., Estrada, M., Woodcock, A., \& Chance, R. C., Sustaining optimal motivation: A longitudinal analysis of interventions to broaden participation of underrepresented students in STEM. Journal of Educational Psychology, 2013. 105(1): 89-107.

\section{Acknowledgements}

\section{Funding}

This work was supported by the UNCF/BMS EE Just Faculty Fund, Burroughs Wellcome Fund Award Career Awards at the Scientific Interface (CASI), NIH SRP subaward to \#5R25HL106365-12 from the NIH PRIDE Program, BWF Ad Hoc Grant to D.M. and AHJ, and the Ford Foundation to A.H.J.; 1K99GM141449-01 MOSAIC grant to C.P.M. and NSF grant MCB \#2011577I to S.A.M.

Acknowledgments: We would like to thank Heather Beasley for helping make the figure in BioRender.

Competing interests: Authors declare that they have no competing interests.

Data and materials availability: All data are available in the main text or the supplementary materials.

369 Racial minorities (P.E.E.R), 21-MortonD-HSR-SOM-01, Kaiser Foundation Research Institute FWA:

$370 \quad$ FWA00002344

371 Ethics Approval and consent to participate, Yes

372 Consent for publication, Yes

373 Competing Interests, Not Applicable

375 Figure Legends

376 Figure 1. Infographics from the workshop depicting growth or developed mindsets compared to fixed or 377 static mindsets. Someone with a growth mindset tries to work with others, as they understand that success 
378 in STEM is not dependent on one person, whereas those with a fixed mindset can avoid collaborating

379 because they see another person succeeding as themselves losing.

380 Figure 2. Results from pre- and post-workshop evaluations for questions 1-4 on their overall knowledge

381 of graduate school.

382 Figure 3. Results from pre- and post-workshop evaluations for questions 5-10 on how well the workshop

383 prepared the participants for the graduate school application.

384 Figure 4. Results from pre- and post-workshop evaluations for questions 11-15 on overall preparedness

385 for starting graduate school.

386 Table 1. Overview and general structure of graduate school.

387 Table 2. Pre- and post- workshop evaluations. 


\section{What kind of mindset do you have?}
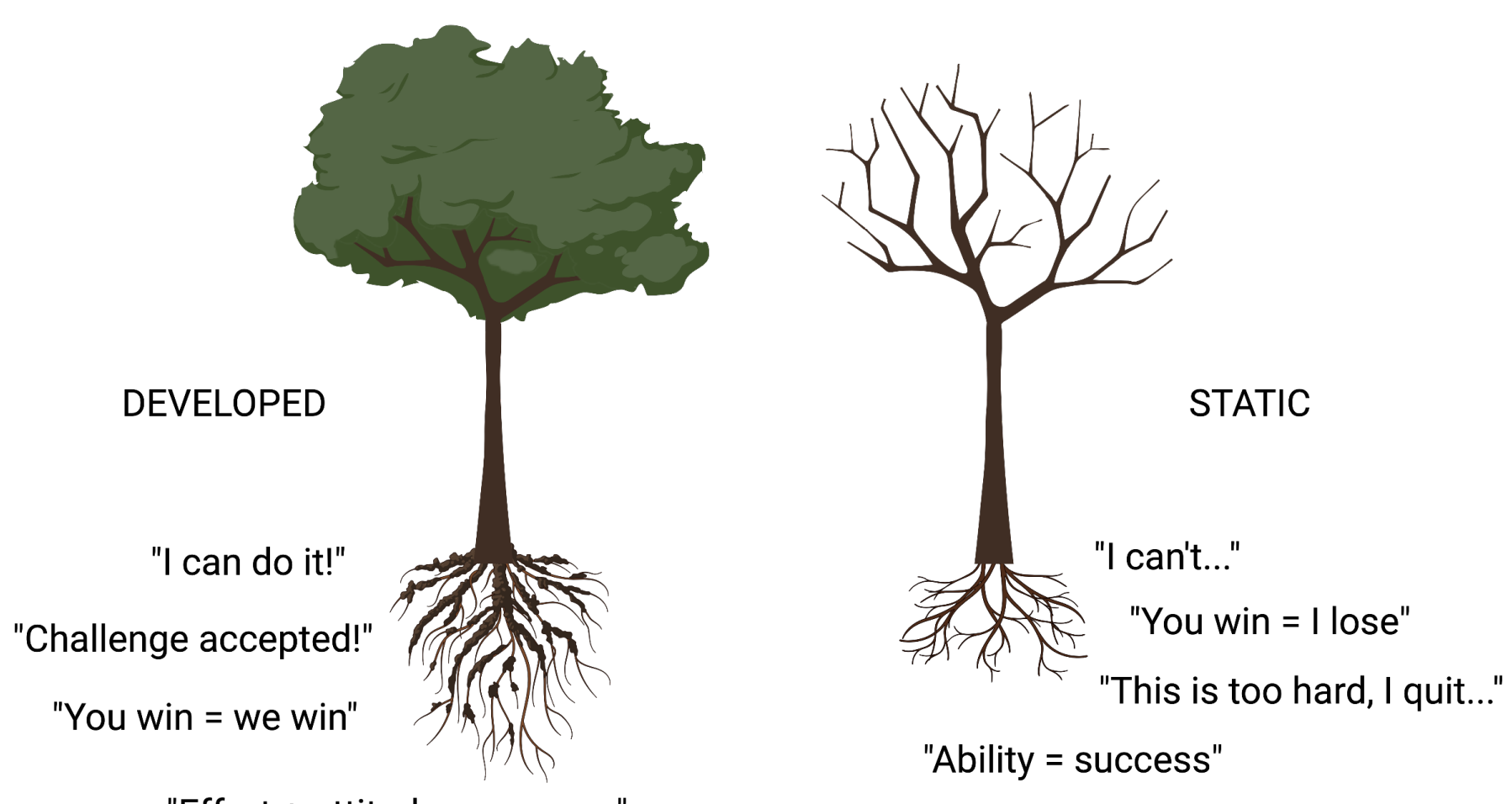

"Effort + attitude = success"

"Ability = success"

\section{Figure 1.}


A.

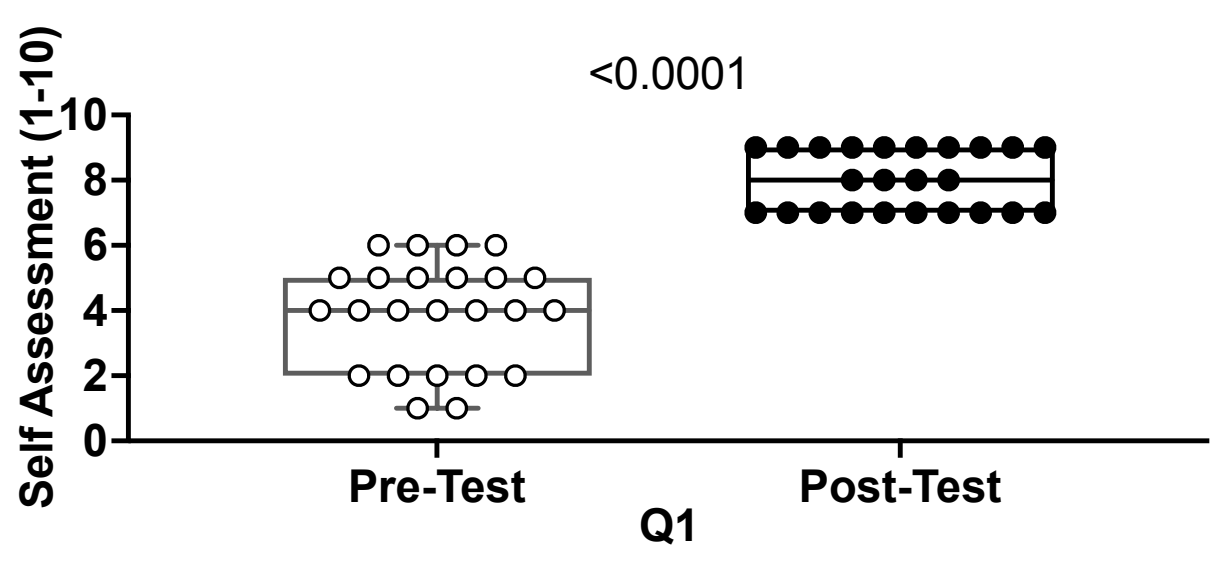

B.
음
$<0.0001$

$\stackrel{5}{5} 10$

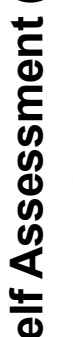

D.
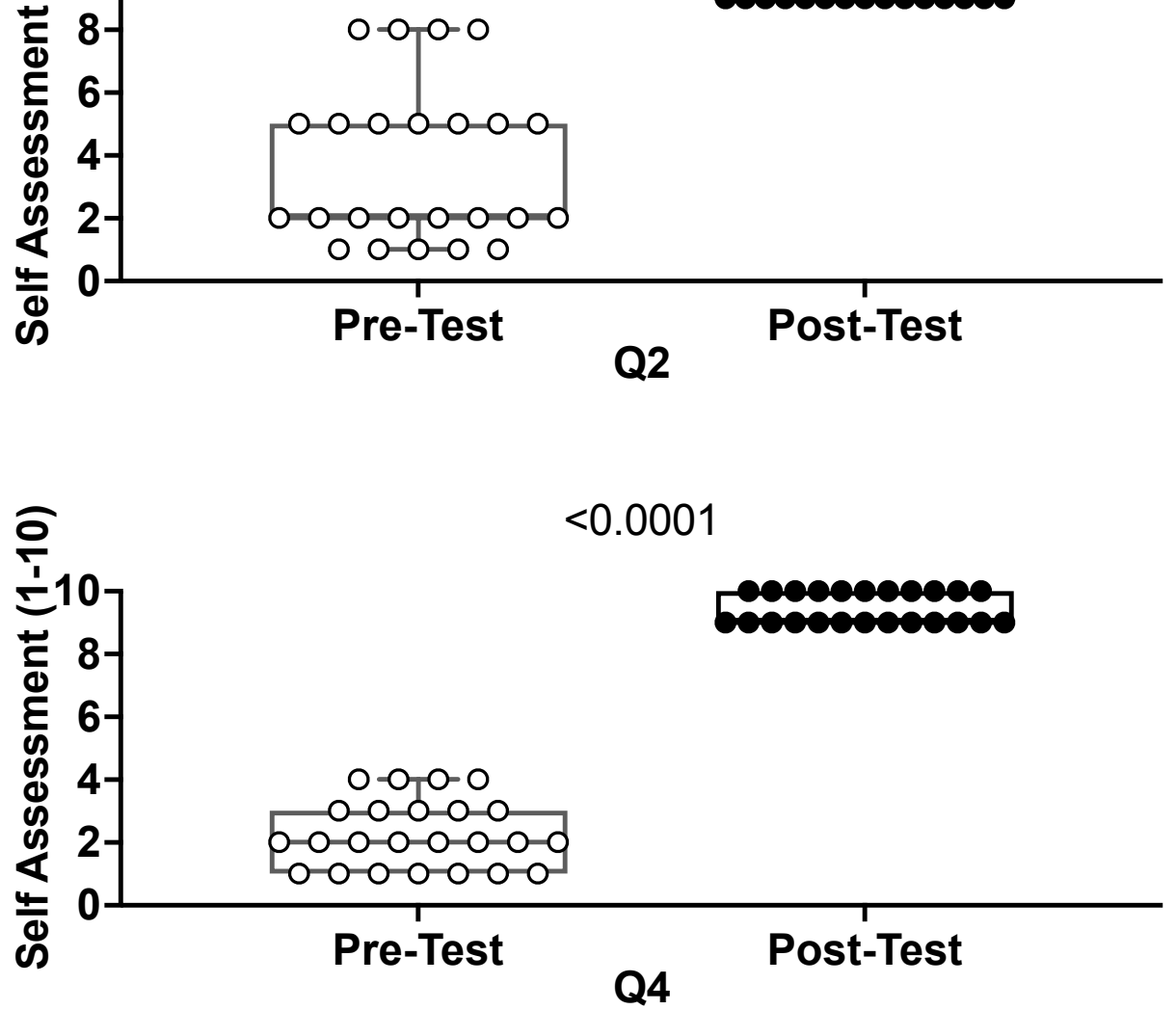

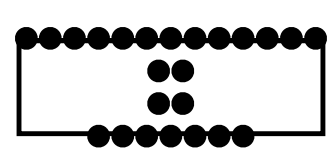

Post-Test
올

000000000000 000000

Pre-Test

Q3 
A.

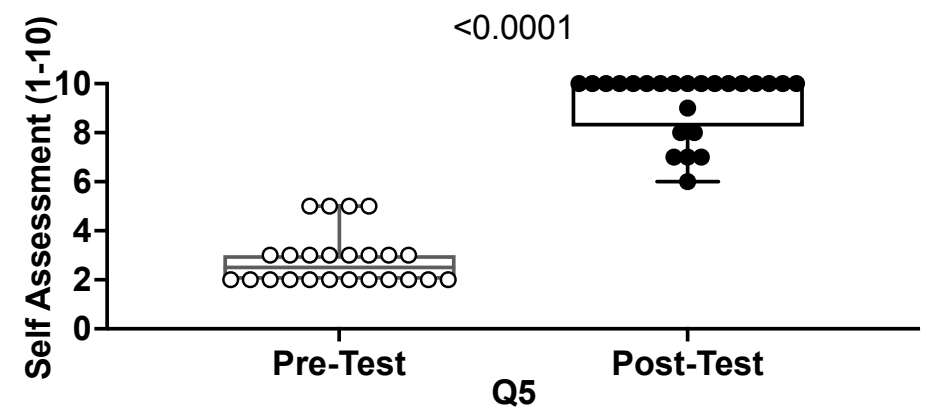

C.

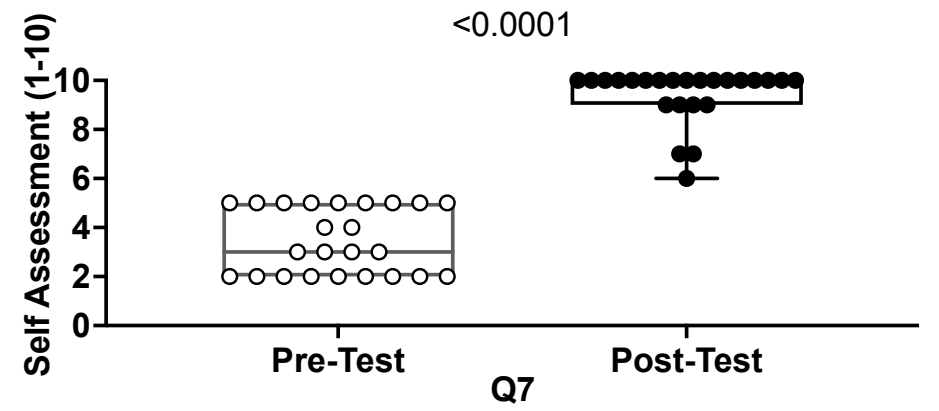

E

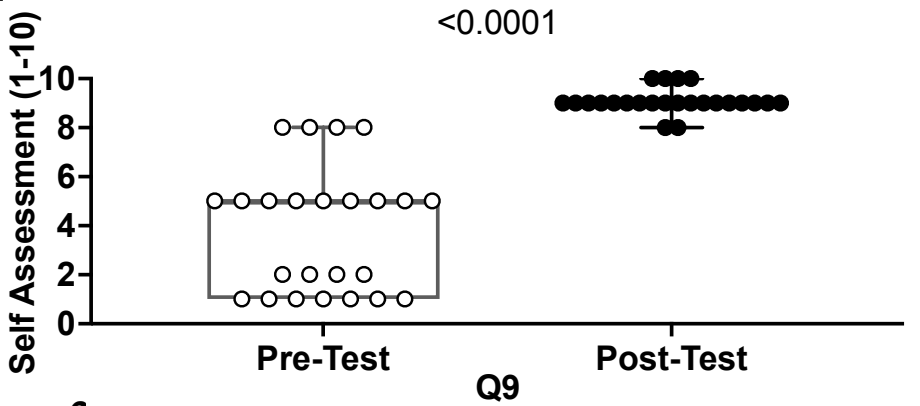

B.

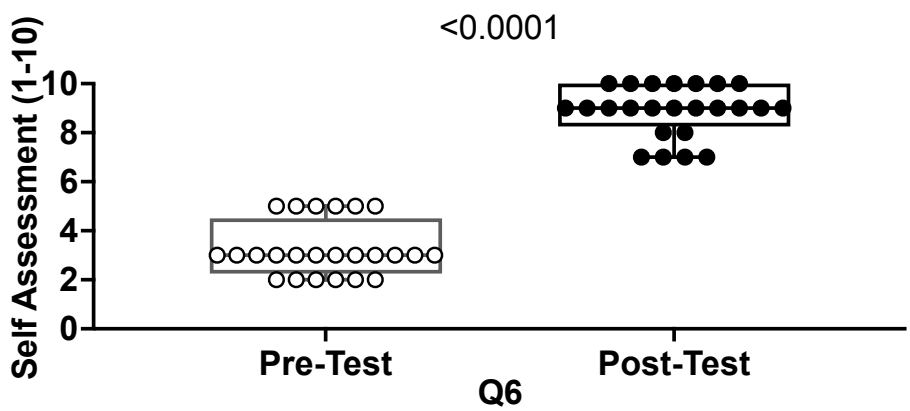

D.

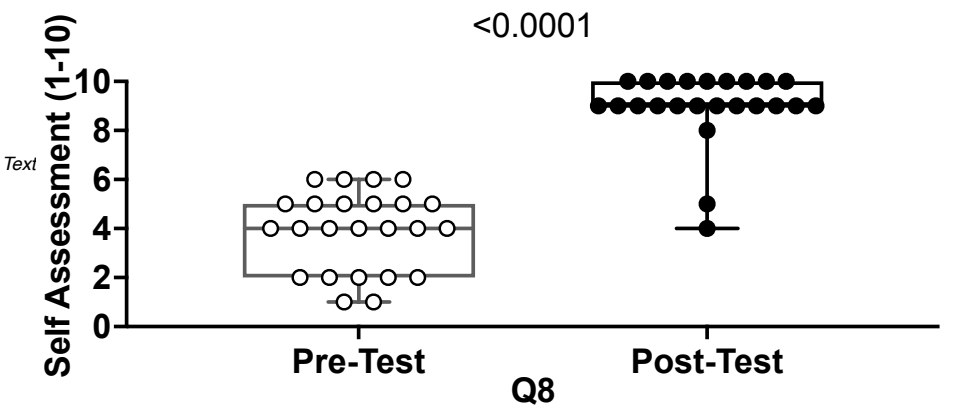

F.

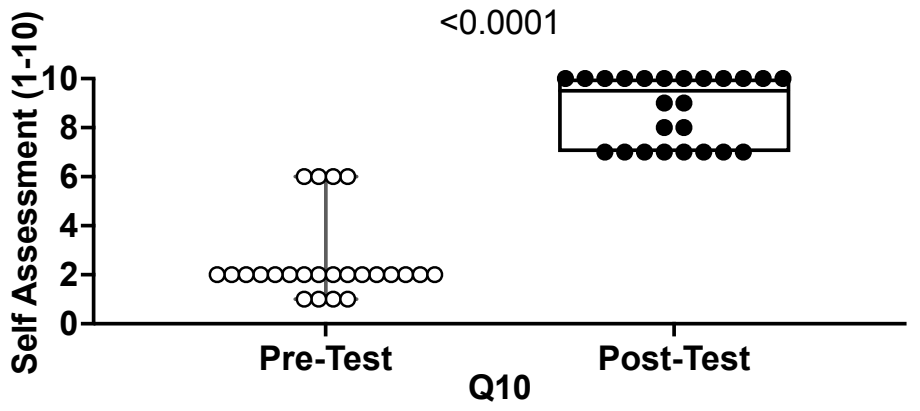


A.

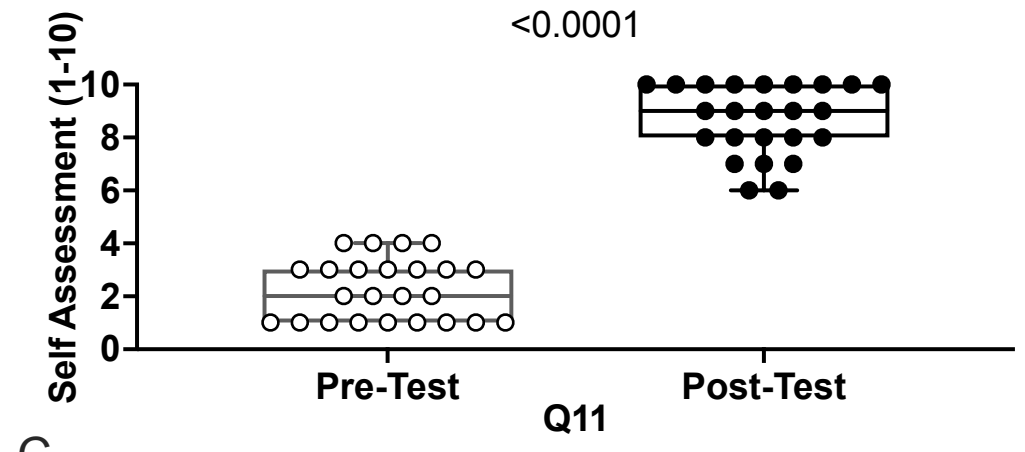

C.

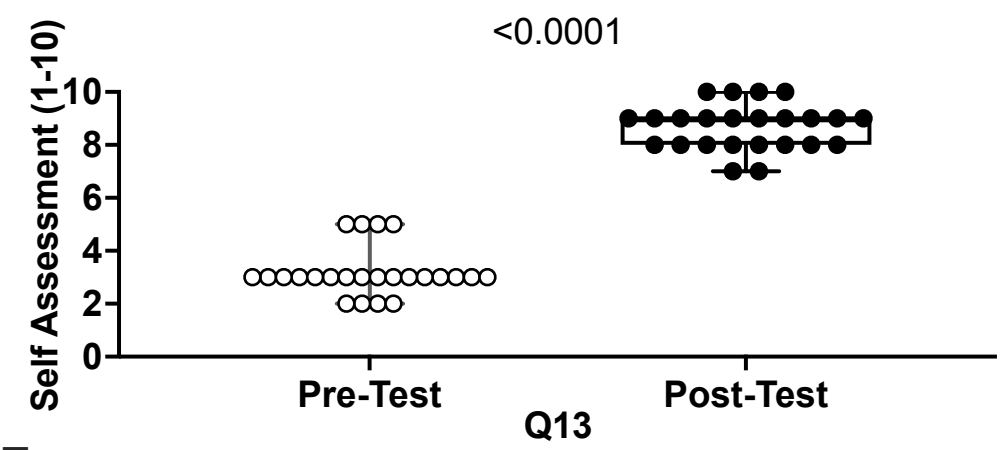

E.

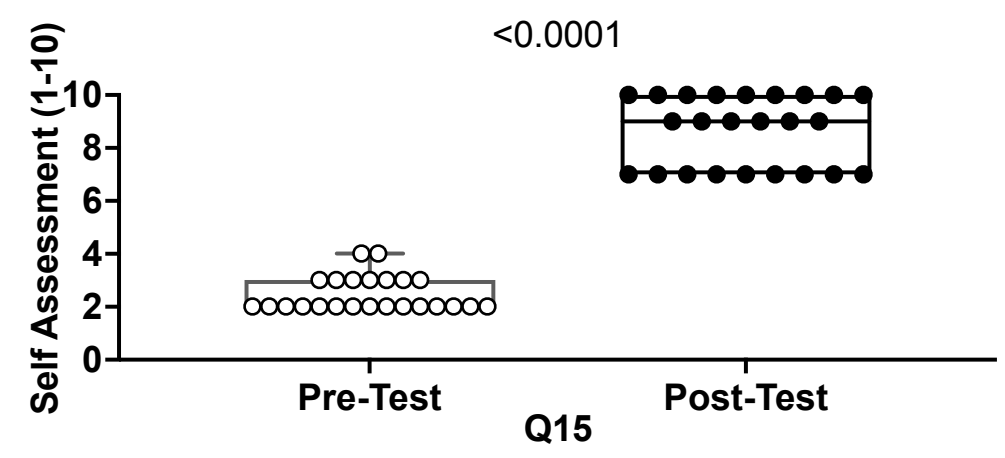

B.

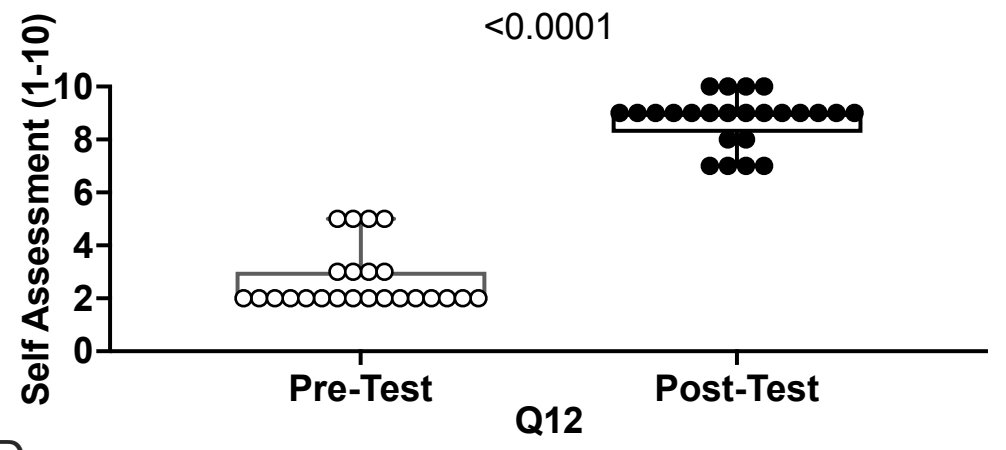

D.

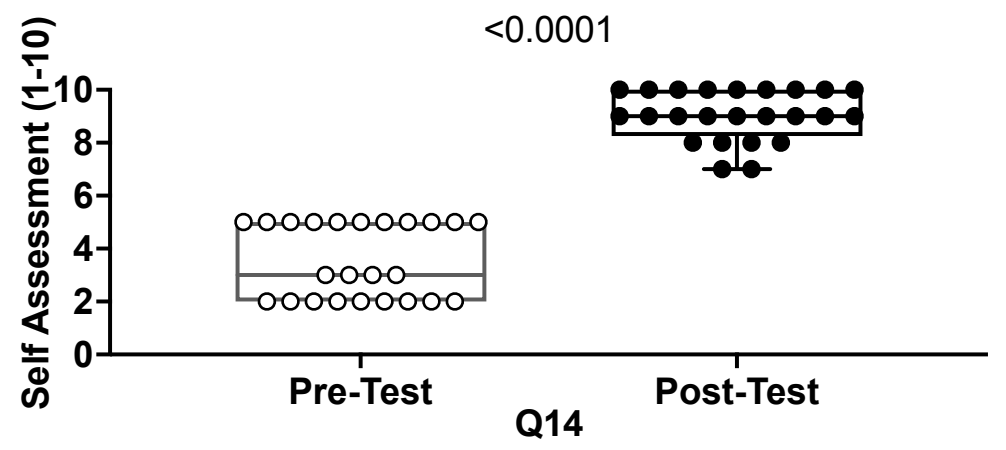


General Program Coursework and Laboratory Rotations

Specialized Coursework and Qualifying Exam

3

Career Development and Grant Writing

4

Collaboration and Publications

$5+$

Next Career Steps and Graduation

Table 1. 


\section{Pre-Test}

1. Do you think the presentation will keep you well informed on a scale of 1 to 10 ?

2. How much do you know about graduate school or professional school on a scale of 1 to 10 ?

3. How well are you prepared for graduate school before this talk on a scale of 1 to 10 ?

4. How well do you think the presentation will better prepare you for graduate school on a scale of 1 to 10 ?

5. How well do you think the talk will improve your personal statement on a scale of 1 to 10 ?

6. How well do you think the talk will improve your choice for letters of recommendation on a scale of 1 to 10 ?

7. How well do you think the talk will improve your confidence to apply to a professional or graduate school ona 1 to 10 scale?

8. How well do you think the talk will improve your studying for the GRE on a scale of 1 to 10 ?

9. How well do you think this talk will improve your networking skills from 1 to 10 ?

10. How well do you think the talk will improve your understanding of why you need to apply to summer internships on a 1 to 10 scale?

11. How well do yo think the talk will improve your time management and stress management on a 1 to 10 scale?

12. How do you think the talk will improve your interview questions on a 1 to 10 scale?

13. How do you think the talk will improve your understanding of what a support team really does on a 1 to 10 scale?

14. How do you think the talk will improve your verbal and non-verbal communication on a 1 to 10 scale?

15. How do you think the talk will improve your learning styles, love langueage, and personality on a 1 to 10 scale?

\section{Post-Test}

1. Do you think the presentation will keep you well informed on a scale of 1 to 10 ?

2. How much do you know about graduate school or professional school on a scale of 1 to 10 ?

3. How well are you prepared for graduate school before this talk on a scale of 1 to 10 ?

4. How well do you think the presentation will better prepare you for graduate school on a scale of 1 to 10 ?

5. How well do you think the talk will improve your personal statement on a scale of 1 to 10 ?

6. How well do you think the talk will improve your choice for letters of recommendation on a scale of 1 to 10 ?

7. How well do you think the talk will improve your confidence to apply to a professional or graduate school ona 1 to 10 scale?

8. How well do you think the talk will improve your studying for the GRE on a scale of 1 to 10 ?

9. How well do you think this talk will improve your networking skills from 1 to 10 ?

0 . How well do you think the talk will improve your understanding of why you need to apply to summer internships on a 1 to 10 scale?

11. How well do yo think the talk will improve your time management and stress management on a 1 to 10 scale?

12. How do you think the talk will improve your interview questions on a 1 to 10 scale?

13. How do you think the talk will improve your understanding of what a support team really does on a 1 to 10 scale?

14. How do you think the talk will improve your verbal and non-verbal communication on a 1 to 10 scale?

15. How do you think the talk will improve your learning styles, love langueage, and personality on a 1 to 10 scale?

\section{Table 2.}

